\title{
Intakes and sources of dietary sugars in Irish pre-school children aged 1-4 years
}

\author{
J. Walton ${ }^{1}$, K Evans ${ }^{1}$, L Kehoe ${ }^{1}$, B. A. McNulty ${ }^{2}$, A. P. Nugent ${ }^{2}$ and A. Flynn ${ }^{1}$ \\ ${ }^{1}$ School of Food and Nutritional Sciences, University College Cork, Republic of Ireland and ${ }^{2}$ UCD Institute of Food \\ and Health, University College Dublin, Belfield, Dublin 4, Republic of Ireland
}

Recent dietary guidance from the UK recommends that, for those aged 2 years and over, the average population intake of free sugars should not exceed $5 \%$ of total energy intake $(\% \mathrm{TE})^{(1)}$. 'Free' sugars include all mono- and di-saccharides added to foods by the manufacturer, cook or consumer, plus sugars naturally present in honey, syrups and unsweetened fruit juices ${ }^{(1)}$. The objective of this study was to estimate the intake and sources of dietary sugars (including free sugars) in 1 to 4 year old Irish children using data from the Irish National Pre-School Nutrition Survey 2010-11 (www.iuna.net) which collected detailed food and beverage intake data using a $4 \mathrm{~d}$ weighed food diary. Energy, total sugars and lactose values were derived from the UK Food Composition Tables ${ }^{(2)}$ and product labels. Non-milk sugars were calculated as total sugars minus lactose. Free sugars values were assigned to each food and beverage consumed by adapting a systematic approach used to calculate added sugars content in foods and beverages ${ }^{(3)}$ and guidance from Public Health England on the calculation of free sugars. Mean intakes of total, non-milk and free sugars were calculated via the NCI-method ${ }^{(4)}$ using SAS $\subset$ Enterprise Guide and key sources of free sugars were determined using SPSS v21.0 .

Table 1. Mean daily intakes of total, non-milk and free sugars (g, \%TE) in Irish children aged $1-4$ years

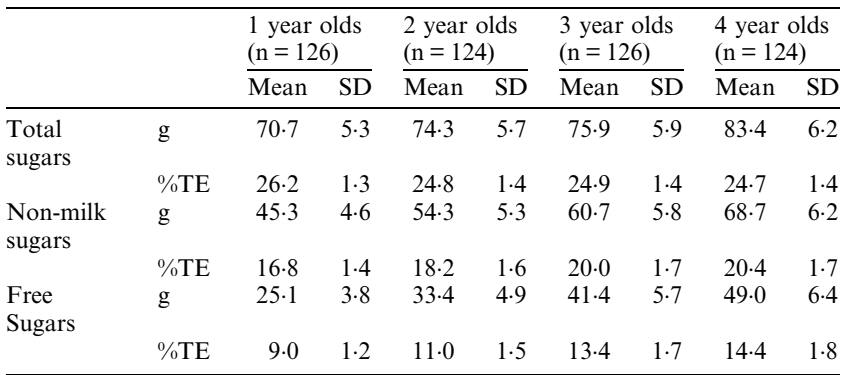

Table 2. \% Contribution of key food-groups to intake of free sugars in Irish children aged $1-4$ years

\begin{tabular}{|c|c|c|c|c|c|c|c|c|}
\hline \multirow{2}{*}{$\begin{array}{l}\text { Age } \\
\text { Food Group }\end{array}$} & \multicolumn{2}{|c|}{$1 \mathrm{y}$} & \multicolumn{2}{|c|}{$2 \mathrm{y}$} & \multicolumn{2}{|c|}{$3 y$} & \multicolumn{2}{|c|}{$4 y$} \\
\hline & g & $\%$ & $\mathrm{~g}$ & $\%$ & $\mathrm{~g}$ & $\%$ & $\mathrm{~g}$ & $\%$ \\
\hline Yoghurts and fromage frais & $6 \cdot 0$ & $31 \cdot 7$ & $5 \cdot 6$ & 17.4 & $6 \cdot 1$ & $15 \cdot 8$ & $6 \cdot 2$ & $13 \cdot 6$ \\
\hline $\begin{array}{l}\text { Fruit juices, smoothies \& } \\
\text { purées }\end{array}$ & $4 \cdot 3$ & $14 \cdot 4$ & $6 \cdot 1$ & $16 \cdot 1$ & $8 \cdot 5$ & $20 \cdot 5$ & 9.4 & $18 \cdot 7$ \\
\hline $\begin{array}{l}\text { unsweetened juices and } \\
\text { smoothies }\end{array}$ & $2 \cdot 1$ & $5 \cdot 8$ & $3 \cdot 6$ & $8 \cdot 7$ & $6 \cdot 0$ & $14 \cdot 2$ & $7 \cdot 2$ & $14 \cdot 0$ \\
\hline fruit purées & $2 \cdot 0$ & $8 \cdot 0$ & $2 \cdot 5$ & $6 \cdot 7$ & $2 \cdot 5$ & $5 \cdot 9$ & $2 \cdot 2$ & $4 \cdot 5$ \\
\hline $\begin{array}{l}\text { Biscuits (incl infant rusks } \\
\text { etc.), cakes \& pastries }\end{array}$ & 3.4 & $14 \cdot 6$ & $4 \cdot 4$ & $12 \cdot 6$ & $4 \cdot 6$ & $12 \cdot 3$ & $6 \cdot 1$ & $13 \cdot 5$ \\
\hline Confectionery & 1.5 & $5 \cdot 2$ & $4 \cdot 0$ & 11.4 & $5 \cdot 3$ & $13 \cdot 2$ & $6 \cdot 3$ & $13 \cdot 2$ \\
\hline chocolate confectionery & $1 \cdot 0$ & $3 \cdot 4$ & $2 \cdot 3$ & $7 \cdot 3$ & $3 \cdot 1$ & 7.9 & $3 \cdot 6$ & 7 \\
\hline $\begin{array}{l}\text { non-chocolate } \\
\text { confectionery }\end{array}$ & $0 \cdot 5$ & $1 \cdot 8$ & 1.7 & $4 \cdot 1$ & $2 \cdot 2$ & $5 \cdot 3$ & $2 \cdot 7$ & 5. \\
\hline Beverages & $2 \cdot 3$ & 7.2 & 5.0 & $12 \cdot 1$ & 4.9 & $10 \cdot 3$ & 6.9 & 12.0 \\
\hline carbonated beverages & $0 \cdot 2$ & 0.5 & $0 \cdot 4$ & 0.9 & $1 \cdot 1$ & $2 \cdot 2$ & $2 \cdot 2$ & 3.6 \\
\hline $\begin{array}{l}\text { squashes and fruit juice } \\
\text { drinks }\end{array}$ & $1 \cdot 4$ & $4 \cdot 5$ & $4 \cdot 4$ & $10 \cdot 6$ & $3 \cdot 8$ & $8 \cdot 0$ & $4 \cdot 6$ & \\
\hline Breakfast cereals & 1.7 & 8.8 & $2 \cdot 3$ & 7.9 & $3 \cdot 1$ & $9 \cdot 1$ & $4 \cdot 1$ & 9.7 \\
\hline $\begin{array}{l}\text { Creams, ice-creams \& chilled } \\
\text { desserts }\end{array}$ & 1.7 & $6 \cdot 1$ & $2 \cdot 2$ & $7 \cdot 5$ & $2 \cdot 5$ & $6 \cdot 7$ & $3 \cdot 3$ & \\
\hline Sugars, syrups \& preserves & 0.8 & 4.5 & 2.4 & $7 \cdot 6$ & 2.6 & $6 \cdot 4$ & $3 \cdot 3$ & 6.7 \\
\hline
\end{tabular}

Intakes of total sugars (\%TE) were similar across ages whereas intakes of non-milk and free sugars (\%TE) increased with increasing age from 1-4 years. Mean intakes of free sugars far exceeded the UK recommended maximum of $5 \%$ of total energy at each age with a number of food categories contributing to these intakes. These findings will be useful in developing dietary strategies to reduce the intakes of free sugars for pre-school children in Ireland.

1 PHE (2015) SACN Carbohydrates and Health Report. London: TSO

2 FSA (2002) The Composition of Foods. Cambridge: Royal Society of Chemistry

3 Louie JCY, Moshtaghian Boylan S et al. (2015) EJCN. 69, 154-161

4 Tooze JA, Kipnis V, Buckman et al. (2010) Stat Med. 29 (27), 2857-68

This research was funded by the Department of Agriculture, Food and the Marine under the Nutridata Project 13F 542; $2014-2016$. 